\title{
Establecimiento y desarrollo de plántulas de Tabebuia rosea (Bignoniaceae) en una selva subcaducifolia manejada de la costa Pacífica de México
}

\author{
Antonio Mora Santacruz ${ }^{1}$, Juan Ignacio Valdez Hernández ${ }^{2}$, Gregorio Ángeles Pérez ${ }^{2}$, \\ Miguel Ángel Musálem Santiago 3 \& Humberto Vaquera Huerta ${ }^{4}$ \\ 1 Departamento de Producción Forestal, Centro Universitario de Ciencias Biológicas y Agropecuarias (CUCBA), \\ Universidad de Guadalajara (UdG). Kilómetro 15.5 Carretera a Nogales, Las Agujas, Nextipac, Apartado postal 129, \\ Zapopan CP 45110, Jalisco, México. Teléfono y Fax: (33) 368202 44; msa19076@cucba.udg.mx \\ 2 Programa Forestal, IRENAT, Colegio de Postgraduados, México \\ 3 Coordinador Nacional Proyecto Sierra Madre. INIFAP, El Horno, Chapingo, México \\ 4 Programa de Estadística. ISEI, Colegio de Postgraduados, México
}

Recibido 13-IV-2003. Corregido 27-V-2005. Aceptado 15-III-2006.

\begin{abstract}
Establishment and development of Tabebuia rosea (Bignoniaceae) seedlings in a semideciduous tropical forest under management, Pacific coast of Mexico. We evaluated the effect of soil "scarification" and vegetation clearing treatments on the natural regeneration and initial development of Tabebuia rosea (Bertold) DC. seedlings in a moderate sized semideciduous tropical forest subjected to wood harvesting on the coast of Jalisco, Mexico. The treatments were applied under "seed" trees, and the number of germinated seedlings and their development were evaluated for nine months. Soil "scarification" promoted seed germination and initial seedling development, while the control of the competing vegetation increased the seedling growth and reduced their mortality. These results should be taken into account for the natural regeneration of this species, after clearing, to improve wood production, and should be incorporated into the silvicultural techniques currently developed in the region. Rev. Biol. Trop. 54 (4): 1215-1225. Epub 2006 Dec. 15.
\end{abstract}

Key words: semideciduous tropical forest, Mexican Pacific coast, natural regeneration, soil scarification, seedling growth and mortality, Tabebuia rosea.

Entre los principales usos de las selvas medianas subcaducifolias en el estado de Jalisco, México, el aprovechamiento maderable es uno de los más importantes, destacando por su valor comercial Tabebuia rosea. Los esquemas de extracción aplicados en este tipo de vegetación se basan en la regeneración natural como el principal fundamento de manejo, sin embargo, uno de los problemas que presentan las áreas bajo aprovechamiento es la escasa regeneración de las especies de interés comercial, entre las que sobresale $T$. rosea. Esta situación ha dado como resultado la disminución de los volúmenes de extracción debido a la falta de incorporación de arbolado joven que sustituya al arbolado maduro que ha sido aprovechado.
Existe una serie de factores que influyen en la regeneración natural y su comprensión requiere conocer los procesos ecológicos que limitan el establecimiento y desarrollo de las plántulas, en especial en aquellas áreas que han sido perturbadas a través de, por ejemplo, los aprovechamientos maderables (Hardwick et al. 1997). El éxito o fracaso de la regeneración natural depende, entre otros, de factores como la producción y dispersión de semillas, así como de las condiciones para la germinación y desarrollo de las plántulas (temperatura, radiación solar y humedad del suelo). Cuando se hace una corta, ésta no es necesariamente suficiente para crear las condiciones apropiadas en la germinación de las semillas y el establecimiento de las plántulas, por lo que en 
ocasiones se hace necesario aplicar tratamientos silviculturales complementarios como la eliminación de residuos de aprovechamiento y el tratamiento al suelo también conocido como escarificación (Hawley y Smith 1972).

Las selvas medianas subcaducifolias se caracterizan por defoliarse en la época seca, acumulándose cantidades importantes de hojarasca en el piso forestal. Esta capa de hojarasca se presenta como una barrera que impide la penetración de las raíces al suelo y limita la germinación de algunas semillas especialmente pequeñas (Hardwick et al. 1997). En condiciones naturales, la apertura de claros es provocada por la caída de árboles, que puede ocasionar un desenraizamiento, exponiendo una superficie del suelo mineral la cual es colonizada por especies arbóreas más rápidamente que aquellas donde la acumulación de residuos es mayor (Hartshorn 1980). El suelo mineral desnudo suele ser un sustrato mucho más favorable, ya que es relativamente estable en cuanto a humedad. La escarificación permite obtener condiciones óptimas para la reproducción de especies con semillas pequeñas (Hawley y Smith 1972).

Además, la competencia de la vegetación rastrera es generalmente un factor importante. En un bosque denso, estas plantas son normalmente escasas, pero después de las cortas y apertura de claros, llegan a cubrir el suelo por completo, extendiéndose a veces tan velozmente que la regeneración de las otras especies no puede prosperar. También las enredaderas y daños mecánicos producidos por la fauna silvestre, así como la caída de ramas y árboles, pueden causar pérdidas graves (Lamprecht 1990). Por tanto, el control de la vegetación competidora mejora la supervivencia y el crecimiento de las plántulas, ya que las malezas compiten por agua, nutrientes y luz en el espacio de crecimiento de una especie (Oliver y Larson 1990). De esta manera, al liberar las plántulas de la competencia se mejora la disponibilidad de recursos, lo que se traduce en una mayor supervivencia y crecimiento inicial, logrando un mejor establecimiento de la nueva masa (Lowery et al. 1993).
En la costa de Jalisco, no se dispone de información acerca de la respuesta de las especies de importancia maderable a tratamientos al suelo y a la vegetación para promover su establecimiento. Por lo que, el presente trabajo tiene como objetivo determinar el efecto que tienen los tratamientos al suelo y a la vegetación competidora sobre el establecimiento y desarrollo de plántulas producto de la regeneración natural de $T$. rosea.

\section{MATERIALES Y MÉTODOS}

Área de estudio: El trabajo se realizó en el predio "El Laurel", municipio de Tomatlán, Jalisco, México (19 $58^{\circ} 47.5^{\prime \prime} \mathrm{N}, 105^{\circ} 04^{\prime} 46.7^{\prime}$ " W). La topografía es accidentada y la altitud oscila entre 200 a $600 \mathrm{msnm}$. La temperatura media anual es de $26{ }^{\circ} \mathrm{C}$ y la precipitación media anual de $1300 \mathrm{~mm}$, con una temporada seca de siete meses bien definida (noviembre a mayo). La vegetación se clasifica como selva mediana subcaducifolia (Miranda y Hernández 1963). La altura del dosel superior oscila entre 20 y 25 m; las especies arbóreas más abundantes son: Brosimum alicastrum Sw. (Moraceae), Hura polyandra Baill (Euphorbiaceae), T. rosea (Bignoneaceae), Bursera simaruba (L.) Sarg. (Burseraceae), Lysiloma acapulcensis (Kunth) Benth. (Fabaceae), Enterolobium cyclocarpum (Jacq.) Griseb. (Fabaceae), Ficus sp. (Moraceae), Astronium graveolens Jacq. (Anacardiaceae), Roseodendron donnell-smithii Rose (Bignoneaceae), Psidium sartorianum (O. Berg) Nied. (Myrtaceae) y Annona squamosa L. (Annonaceae) (Anónimo 1994). El aprovechamiento maderable se ha realizado por 15 años en este predio, con base en programas de manejo forestal, aplicándose cortas de selección (entresaca), extrayéndose arbolado sobremaduro de especies comerciales. El pastoreo extensivo es otro uso significativo de la selva.

Descripción de la superficie experimental: El experimento se ubicó en un rodal de aproximadamente 1.5 ha, donde en 1987 se aplicó una corta de refinamiento eliminando 
las especies no comerciales, dejando en pie solo algunos árboles de $H$. polyandra, $T$. rosea y $E$. cyclocarpum (especies comerciales). El perímetro del área está cercado con alambre de púas para prevenir la entrada del ganado. El rodal está conformado por dos estratos: uno superior compuesto en su mayoría por árboles adultos de especies comerciales, y otro inferior que consiste principalmente de especies arbustivas y herbáceas, las cuales son periódicamente eliminadas con el propósito de favorecer el crecimiento de especies con valor comercial.

Efecto de los tratamientos al suelo y vegetación en el establecimiento de la regeneración natural: Se escogieron árboles muestra o "semilleros", adultos y en edad reproductiva (45-55 cm de DAP, 20-25 m de altura), separados entre sí de tal manera que no hubiera sobreposición entre sus copas, debajo de las cuales se aplicaron los siguientes tratamientos: a) Remoción de suelo, una vez eliminada y retirada la vegetación herbácea y arbustiva, se revolvió el suelo con una herramienta manual, incorporando la materia orgánica (hojarasca) hasta dejar expuesto el suelo mineral, también se extrajeron con todo y raíz los pequeños tocones de los arbustos; y b) Roza de vegetación, eliminación con machete de la vegetación herbácea y arbustiva, retirando los residuos, pero sin remover el suelo, manteniendo la capa de hojarasca y ramillas pequeñas.

La forma de las parcelas fue rectangular, de $2 \mathrm{~m}$ de ancho por una longitud igual a dos veces el tamaño de la copa de cada árbol "semillero", partiendo del fuste hacia el límite exterior de la copa; es decir, sobrepasando la zona de goteo en el doble de su tamaño, esto con el propósito de ampliar el área potencial de captación de las semillas. En promedio las parcelas tuvieron una longitud de $12.5 \mathrm{~m}$ y una superficie de $24 \mathrm{~m}^{2}$. La orientación de todas las parcelas siguió rumbos francos trazados con brújula: Norte, Sur, Este y Oeste, tomando como punto de partida el fuste de cada árbol semillero.

En total fueron cinco árboles semilleros con 20 parcelas experimentales, de las cuales 12 correspondieron al tratamiento de remoción de suelo y las ocho restantes al tratamiento roza de vegetación. La toma de datos en campo se realizó al final de la cuarta semana (julio del 2000), registrándose el número de plántulas emergidas: cotiledones visibles por arriba de la superficie del suelo.

\section{Influencia de los tratamientos al suelo y vegetación sobre el desarrollo inicial de plántulas}

Crecimiento: Para su evaluación se utilizaron subparcelas de 2 × 2 m ubicadas dentro de las parcelas ya establecidas bajo los árboles semilleros. Los tratamientos evaluados fueron: a) Remoción de suelo con deshierbe y b) Roza de vegetación con deshierbe, los cuales son los mismos a los descritos anteriormente, con la diferencia que a estos últimos se les aplicó deshierbe. El deshierbe se realizó en forma manual, manteniendo las subparcelas limpias de maleza.

Todas las plántulas fueron marcadas y las evaluaciones se realizaron en forma mensual durante nueve meses (noviembre 2000-julio 2001). Las variables que se midieron fueron altura $(\mathrm{cm})$, diámetro al cuello de la raíz $(\mathrm{mm})$ y número de hojas.

Vegetación competidora: El control de esta vegetación se evaluó en las subparcelas de 2 × 2 m descritas anteriormente y bajo los siguientes tratamientos: a) Remoción de suelo con deshierbe (Remo c/d), b) Roza de vegetación con deshierbe (Roza c/d), c) Remoción de suelo sin deshierbe (Remo s/d) y d) Roza de vegetación sin deshierbe (Roza s/d).

Las variables evaluadas fueron altura total $(\mathrm{cm})$, diámetro al cuello de la raíz $(\mathrm{mm})$ y número de hojas. La toma de datos se efectuó en tres periodos: marzo, mayo y julio del 2001, este último al inicio de las lluvias. Lo anterior se debió a que en las subparcelas donde no se aplicó deshierbe, la maleza creció tan rápida y abundantemente que no permitió hacer mediciones sin alterar la cobertura original, por lo que se decidió esperar hasta que la maleza disminuyera para poder medir las plántulas. 
Mortalidad de plántulas: El periodo de evaluación de la mortalidad comprendió 12 meses, inició con la aplicación del deshierbe en agosto del 2000 y concluyó en julio del 2001. Los tratamientos de remoción y roza con deshierbe se evaluaron cada mes (agosto del 2000 a julio del 2001), mientras que en los tratamientos de remoción y roza sin deshierbe solamente se realizaron tres evaluaciones: marzo, mayo y julio del 2001. Esto con el propósito de no alterar la cobertura original, como se describió anteriormente.

Análisis de datos: Con la finalidad de detectar posibles diferencias significativas $(p<0.05)$ entre los tratamientos al suelo y vegetación sobre el establecimiento de la regeneración natural y desarrollo inicial de plántulas, se llevaron a cabo análisis de varianza (ANDEVA) bajo un diseño experimental completamente al azar (Infante y Zárate 1990). Para evaluar el efecto de la vegetación competidora sobre el desarrollo inicial de plántulas también se realizaron análisis de varianza, complementados por pruebas de comparación de medias (Tukey, $\mathrm{p}<0.05)$ de acuerdo con Cochran (1998). Los análisis estadísticos anteriores se efectuaron con el paquete S-Plus 2000 (Anónimo 1999).

\section{RESULTADOS}

\section{Efecto de los tratamientos al suelo y vegetación en el establecimiento de la regeneración natural}

La mayor cantidad de plántulas por unidad de superficie correspondió al tratamiento remoción de suelo con 5.16 plántulas por $\mathrm{m}^{2}$, mientras que el tratamiento roza de vegetación tuvo solo 0.82 plántulas por $\mathrm{m}^{2}$ (Fig. 1).

Los resultados anteriores permiten establecer que el tratamiento remoción de suelo tuvo un efecto positivo sobre la densidad de plántulas emergidas (ANDEVA, $\mathrm{F}=12.35, \mathrm{p}<0.01$ ).

\section{Influencia de los tratamientos al suelo y vegetación sobre el desarrollo inicial de plántulas}

Crecimiento: El periodo de evaluación abarcó de noviembre del 2000 (temporada seca) a julio del 2001 (temporada húmeda). De las 353 plántulas marcadas, 303 correspondieron al tratamiento remoción de suelo y 50 al de roza de vegetación.

El crecimiento en altura de las plántulas fue significativamente (ANDEVA, $\mathrm{p}<0.01$ para los meses de noviembre $\mathrm{F}=10.10$, diciembre $\mathrm{F}=7.83$, abril $\mathrm{F}=7.83$ y julio $\mathrm{F}=9.09, \mathrm{y} \mathrm{p}<0.05$ para enero $\mathrm{F}=7.40$, febrero $\mathrm{F}=7.35$, marzo $\mathrm{F}=7.48$, mayo $\mathrm{F}=6.92$ y junio $\mathrm{F}=6.69$ ) mayor en el tratamiento remoción de suelo en comparación con el tratamiento roza de la vegetación para cada fecha de medición (Fig. 2).

La altura promedio de las plántulas al final del periodo de evaluación (julio 2001) fue

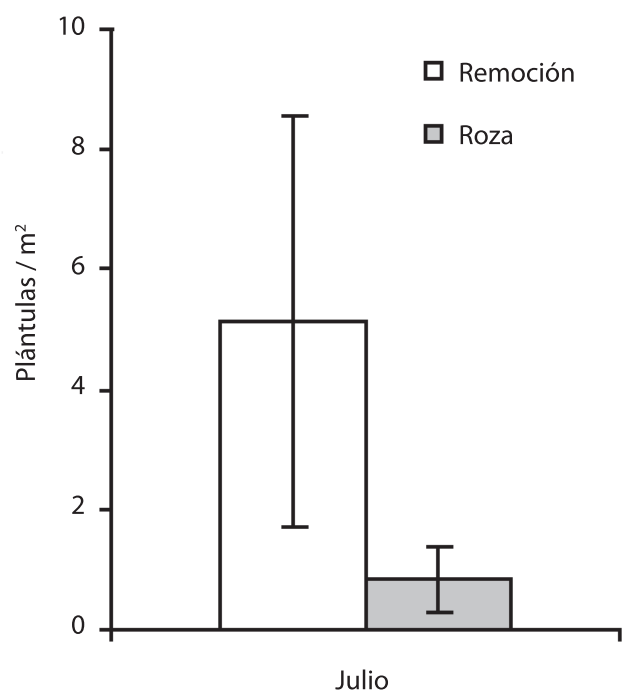

Fig. 1. Número promedio de plántulas de T. rosea después de aplicados los tratamientos. Las líneas verticales representan la desviación estándar.

Fig. 1. Average number of T. rosea seedlings after the application of treatments. Vertical lines represent standard deviation. 


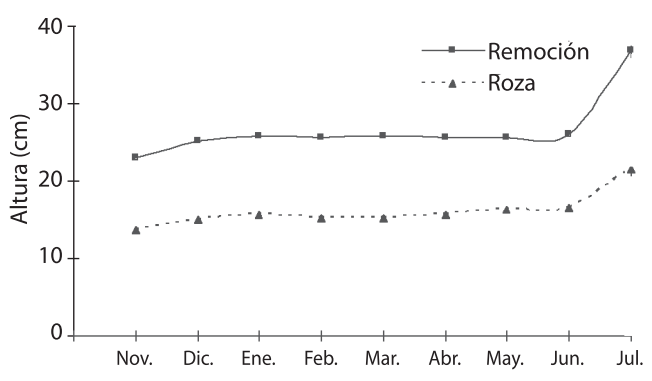

Fig. 2. Crecimiento promedio en altura de plántulas de $T$. rosea bajo los tratamientos remoción de suelo $(\mathrm{n}=303)$ y roza de la vegetación $(n=50)$.

Fig. 2. Average growth in height of T. rosea seedlings for soil scarification $(n=303)$ and vegetation cutting treatments $(n=50)$.

$58.3 \%$ mayor para el tratamiento remoción de suelo $(36.9 \mathrm{~cm})$ en comparación con el tratamiento roza de la vegetación $(21.5 \mathrm{~cm})$.

El crecimiento en diámetro de las plántulas fue significativamente (ANDEVA, $\mathrm{p}<0.01$ para los meses de noviembre $\mathrm{F}=19.48$, diciembre $\mathrm{F}=$ 9.92 y enero $\mathrm{F}=8.37$; $y$ de $\mathrm{p}<0.05$ para febrero $\mathrm{F}=4.25$, marzo $\mathrm{F}=6.09$, abril $\mathrm{F}=5.68$, mayo $\mathrm{F}=4.40$, junio $\mathrm{F}=4.26$ y julio $\mathrm{F}=5.59$ ) mayor en el tratamiento remoción de suelo en comparación con el tratamiento roza de la vegetación para cada fecha de medición (Fig. 3).

El diámetro promedio de las plántulas al final del periodo de evaluación (julio 2001) fue $70.3 \%$ mayor para el tratamiento remoción de suelo $(10.76 \mathrm{~mm})$ en comparación con el tratamiento roza de la vegetación $(7.57 \mathrm{~mm})$.

Sin embargo, en ambos tratamientos las plántulas tuvieron una forma cónica del tallo: grueso en la base y ahusándose hacia la punta o yema apical, y presentaron una contracción en mayo: época más seca del año (Fig. 3).

La cantidad de hojas generada durante el periodo de evaluación, bajo los tratamientos descritos, fue muy similar (Fig. 4). El análisis de varianza del número de hojas mostró diferencias significativas entre tratamientos (ANDEVA, $\mathrm{p}<0.05$ ) sólo para la fecha inicial de muestreo (noviembre $\mathrm{F}=5.85$ ), mientras que para los meses restantes no hubo diferencias significativas.

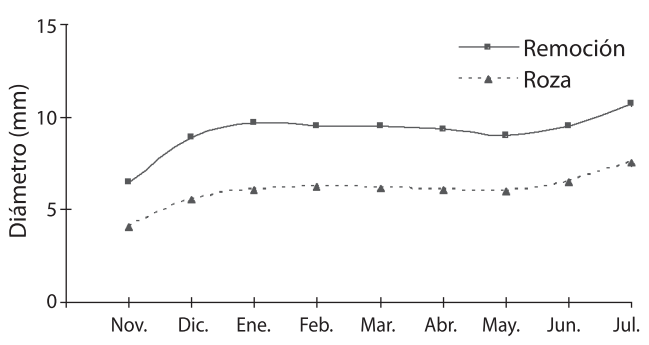

Fig. 3. Crecimiento promedio en diámetro de plántulas de T. rosea bajo los tratamientos remoción de suelo $(n=303)$ y roza de la vegetación $(n=50)$.

Fig. 3. Average growth in diameter of $T$. rosea seedlings for soil scarification $(n=303)$ and vegetation cutting treatments $(n=50)$.

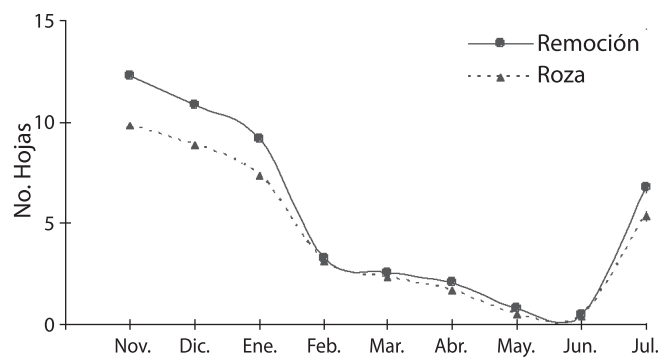

Fig. 4. Número promedio de hojas en plántulas de $T$. rosea bajo los tratamientos remoción de suelo $(n=303)$ y roza de la vegetación $(n=50)$.

Fig. 4. Mean number of leaves in T. rosea seedlings for soil scarification $(n=303)$ and vegetation cutting treatments $(n=50)$.

Vegetación competidora: De 565 plántulas evaluadas, 303 fueron para remoción de suelo con deshierbe, 208 para remoción de suelo sin deshierbe, 50 para roza de vegetación con deshierbe y cuatro para roza de vegetación sin deshierbe. El mayor crecimiento en altura se presentó en la condición de remoción de suelo con deshierbe; sin embargo, el crecimiento en altura entre los tratamientos de roza con deshierbe y remoción sin deshierbe fue muy similar en los tres periodos de evaluación (Fig. 5A). Lo mismo ocurrió para el número de hojas en los tratamientos de remoción con deshierbe y roza con deshierbe (Fig. 5C), pero no así con el crecimiento en diámetro donde el tratamiento remoción con deshierbe fue superior (Fig. 5B). 

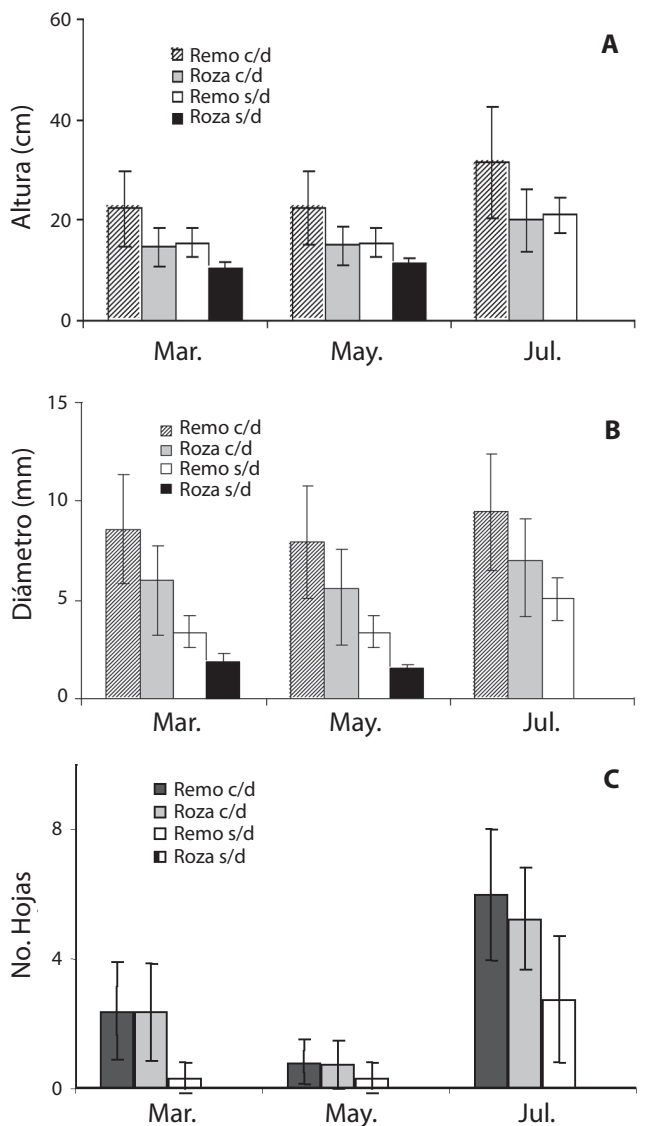

Fig. 5. Crecimiento en altura (A.), diámetro (B.) y número de hojas (C.) en plántulas de T. rosea bajo diferentes tratamientos y en tres fechas de muestreo (2001). Las líneas verticales representan la desviación estándar.

Fig. 5. Growth in height (A.), diameter (B.) and number of leaves (C.) in T. rosea seedlings for different treatments and in three sampling dates (2001). Vertical lines represent standard deviation.

Los resultados del análisis de varianza para el crecimiento en altura de las plántulas manifestaron diferencias significativas (marzo $\mathrm{F}=8.31, \mathrm{p}<0.01 ;$ mayo $\mathrm{F}=8.04, \mathrm{p}<0.01$; julio $\mathrm{F}=7.90, \mathrm{p}<0.01)$. Al menos un tratamiento fue diferente en cada uno de los periodos de evaluación. Se utilizó la prueba de Tukey para comparar las medias (Cuadro 1). Los tratamientos roza con deshierbe y remoción sin deshierbe comparten similitud estadística en los tres periodos de evaluación. El tratamiento que presentó las plántulas con alturas significativamente mayores a los demás tratamientos fue el de remoción con deshierbe.

Los resultados del análisis de varianza para el crecimiento en diámetro muestran evidencias altamente significativas de que existen diferencias entre tratamientos en todas las fechas de muestreo (marzo $\mathrm{F}=24.68, \mathrm{p}<0.01$; mayo $\mathrm{F}=21.63, \mathrm{p}<0.01$; julio $\mathrm{F}=11.18, \mathrm{p}<0.01)$. La prueba de Tukey (Cuadro 1) muestra que el tratamiento roza sin deshierbe fue el que presentó valores menores de diámetro, seguido por el tratamiento remoción sin deshierbe; y el que mayores valores registró fue el de remoción con deshierbe.

Los resultados del análisis de varianza muestran diferencias altamente significativas entre tratamientos en el número de hojas de las plántulas para dos fechas de medición (marzo $\mathrm{F}=12.21, \mathrm{p}<0.01$; julio $\mathrm{F}=9.48, \mathrm{p}<0.01)$. La prueba de Tukey (Cuadro 1) mostró que los tratamientos remoción y roza ambos sin deshierbe fueron los que presentaron valores significativamente menores en número de hojas para tales fechas de evaluación, en comparación con los tratamientos remoción y roza ambos con deshierbe, que fueron estadísticamente similares entre sí.

En general, los mayores crecimientos de las plántulas en altura, diámetro y número de hojas, se presentaron en aquellas desarrolladas bajo el tratamiento remoción de suelo con deshierbe; es decir, que crecieron libres de malezas.

Mortalidad: Se observó una clara tendencia de la mortalidad de plántulas hacia aquellos tratamientos donde no se aplicó deshierbe (Cuadro 2).

En la última evaluación (julio del 2001), al principio de la temporada de lluvias, se encontró que los mayores porcentajes de mortalidad correspondieron al tratamiento roza sin deshierbe con el $100 \%$, seguido por remoción sin deshierbe con $87 \%$, roza con deshierbe con $55 \%$ y el que menor proporción presentó fue remoción con deshierbe con $34 \%$. Las plántulas que crecieron bajo la maleza se mostraron débiles y raquíticas, por lo que la mayoría de 
CUADRO 1

Comparación de medias entre tratamientos para crecimiento en altura, diámetro y número de hojas de plántulas de T. rosea en diferentes fechas de muestreo

TABLE 1

Means comparison between treatments for growth in height, diameter and number of leaves in T. rosea seedlings in different sampling dates

\begin{tabular}{|c|c|c|c|c|c|}
\hline \multirow{2}{*}{ Variable } & \multirow{2}{*}{ Fecha } & \multicolumn{4}{|c|}{ Tratamientos } \\
\hline & & $\mathrm{T} 1$ & $\mathrm{~T} 2$ & $\mathrm{~T} 3$ & $\mathrm{~T} 4$ \\
\hline \multirow[t]{3}{*}{ Altura $(\mathrm{cm})$} & Marzo & $22.37 \mathrm{a}$ & $14.70 \mathrm{~b}$ & $15.43 \mathrm{~b}$ & $10.50 \mathrm{~b}$ \\
\hline & Mayo & $22.39 \mathrm{a}$ & $15.03 \mathrm{~b}$ & $15.46 \mathrm{~b}$ & $11.33 \mathrm{~b}$ \\
\hline & Julio & $31.57 \mathrm{a}$ & $19.96 \mathrm{~b}$ & $21.04 \mathrm{~b}$ & $0.0 \mathrm{c}$ \\
\hline \multirow[t]{3}{*}{ Diámetro (mm) } & Marzo & $8.56 \mathrm{a}$ & $5.94 \mathrm{~b}$ & $3.38 \mathrm{c}$ & $1.84 \mathrm{c}$ \\
\hline & Mayo & $7.95 \mathrm{a}$ & $5.62 \mathrm{ab}$ & $3.38 \mathrm{bc}$ & $1.57 \mathrm{c}$ \\
\hline & Julio & $9.68 \mathrm{a}$ & $7.02 \mathrm{ab}$ & $5.07 \mathrm{~b}$ & $0.0 \mathrm{c}$ \\
\hline \multirow[t]{3}{*}{ Número de hojas } & Marzo & $2.38 \mathrm{a}$ & $2.36 \mathrm{a}$ & $0.34 \mathrm{~b}$ & $0.0 \mathrm{~b}$ \\
\hline & Mayo & $0.82 \mathrm{a}$ & $0.73 \mathrm{a}$ & $0.34 \mathrm{a}$ & $0.0 \mathrm{a}$ \\
\hline & Julio & $5.97 \mathrm{a}$ & $5.24 \mathrm{a}$ & $2.75 \mathrm{~b}$ & $0.0 \mathrm{~b}$ \\
\hline
\end{tabular}

Las medias que presentan la misma letra entre columnas no son estadísticamente diferentes $(\mathrm{p} \leq 0.05)$. T1= Remoción de suelo con deshierbe; T2= Roza de vegetación con deshierbe; T3= Remoción de suelo sin deshierbe; T4= Roza de vegetación sin deshierbe.

ellas no logró sobreponerse a las condiciones adversas de competencia.

\section{DISCUSIÓN}

\section{Establecimiento de la regeneración natural}

La emergencia de las plántulas fue mayor en el tratamiento remoción de suelo, ya que se dejó expuesto el suelo mineral, proporcionando una cama semillera donde la radícula de las plántulas alcanzó fácilmente el mismo. Los resultados anteriores son congruentes con los informados por Musálem (1984) para Pinus montezumae y Velázquez (1984) para Pinus hartwegii en un bosque de clima templado de México, así como con los de Karlsson y Örlander (2000) para Pinus sylvestris en Suecia.
Se ha documentado que con la remoción del suelo se incorpora la capa de hojarasca que llega a ser una barrera que impide el contacto de las semillas con el suelo limitando su germinación (Beland et al. 2000), siendo significativamente mayor la germinación en suelo mineral que en suelo mezclado con humus y suelo sin remover. Asimismo, se ha encontrado que la presencia de hojarasca tiene también un efecto negativo en el establecimiento de especies con semillas pequeñas (Molofsky y Augspurger 1992).

No obstante que para algunas especies la capa de hojarasca y de humus en descomposición puede ser una buena cama semillera, debido a su alta capacidad de infiltración de agua, buena aireación y a que favorece el contacto de las partículas del suelo con el agua y la semilla (Kozlowski 2002), muchas plántulas 
CUADRO 2

Valores absolutos de mortalidad en plántulas de T. rosea por tratamiento y en diferentes fechas de muestreo

TABLE 2

Absolute values of mortality in $\mathrm{T}$. rosea seedlings by treatment and in different sampling dates

Fecha

Agosto (2000)
Septiembre
Octubre

Noviembre

Diciembre

Enero (2001)

Febrero

Marzo

Abril

Mayo

Junio

Julio

Total

Mortalidad (\%)

T1

V
772

738

729

725

716

716

712

710

708

703

658

510

510

$\mathrm{T} 1=$ Remoción de suelo con deshierbe, T2= Roza de vegetación con deshierbe, $\mathrm{T} 3=$ Remoción de suelo sin deshierbe, $\mathrm{T} 4=$ Roza de vegetación sin deshierbe, $\mathrm{V}=$ Plántulas vivas, $\mathrm{M}=$ Plántulas muertas.

mueren debido a que sus raíces no logran penetrar dicha capa hasta llegar al suelo mineral (Ahlgren 1974).

Los resultados obtenidos en el presente estudio, y el hecho que algunas especies requieren de suelos desnudos para el establecimiento de sus plántulas (Finegan 1984), se puede establecer que $T$. rosea requiere de suelo mineral para su germinación. La germinación de las semillas de esta especie fue favorecida con la eliminación de hojarasca, ya que debido a su tamaño $(0.0166 \mathrm{~g})$ no tuvo la capacidad para que sus raíces atravesaran esta barrera y alcanzaran el suelo mineral. Estos resultados son similares a lo informado por Macario (1991) quien menciona que si las semillas al caer en la hojarasca logran germinar durante la época húmeda, éstas pueden morir durante la época seca debido a que sus raíces no alcanzan el suelo mineral. En general, la disponibilidad de agua en el suelo mineral es más estable que en la capa de hojarasca (Ángeles y Sakimoto 1999).

\section{Desarrollo inicial de plántulas}

El mayor crecimiento en altura y diámetro de las plántulas de $T$. rosea ocurrió en el tratamiento remoción de suelo. Probablemente con el aflojamiento del suelo las plántulas desarrollaron un mayor sistema radical que permitió un mayor suministro de agua y nutrientes. Sin embargo, aunque no hubo un efecto de los tratamientos sobre el número de hojas, se observó que las plántulas desarrolladas en el tratamiento de remoción de suelo tuvieron hojas de mayor tamaño que aquellas en el tratamiento roza de vegetación. Este factor no se evaluó en la presente investigación pero pudo ejercer un efecto 
positivo sobre el desarrollo de las plántulas, como se manifestó en los análisis anteriores de crecimiento en altura y diámetro.

Asimismo, Hartshorn (1980) y Macario (1991) informan que las plántulas se desarrollan mejor en lugares iluminados y con suelo expuesto, mientras que Karlsson y Örlander (2000) señalan que la escarificación o remoción del suelo mejoran la germinación de las semillas y aumentan el crecimiento de las plántulas.

\section{Efecto de la vegetación competidora}

Los resultados muestran que el crecimiento en altura de $T$. rosea fue menos sensible a la eliminación de la vegetación competidora, al parecer, la competencia de hierbas y arbustos redujo el crecimiento en diámetro más que el crecimiento en altura.

De acuerdo con esto, Ángeles et al. (1997) informan que el incremento en diámetro de Pinus patula (especie de bosque templado) fue mayor donde se tuvo control de la maleza que donde no se tuvo. También mencionan que al existir un mayor espacio disponible, los brinzales sujetos al tratamiento de control de la maleza tuvieron oportunidad de apropiarse de una mayor cantidad de recursos, lo cual se tradujo en un mayor crecimiento radial.

La competencia de las plántulas con hierbas y arbustos se presenta principalmente de dos formas (Holl 1998): a) a nivel de sistema radical, disputando nutrientes y agua; y b) por la luz; en ambos casos se reduce el crecimiento. Augspurger (1984) demostró que algunas especies tropicales crecieron más rápidamente cuando fueron expuestas a altos niveles de luz, lo cual sucede con $T$. rosea considerada como una especie heliófita que requiere luz directa para su desarrollo.

En el presente trabajo, en los tratamientos donde se removió el suelo, el crecimiento de las plántulas de esta especie fue más afectado cuando se aplicó deshierbe, observándose claramente que las plántulas desarrolladas libres de maleza presentaron mayores crecimientos que aquellas donde no se realizó el control de la misma. Estos resultados coinciden con los informados por Uhl (1987), quien señala que la competencia por luz es más importante que la disponibilidad de agua o minerales en el suelo.

Las hierbas y arbustos tuvieron un efecto negativo sobre el desarrollo de las plántulas de $T$. rosea, debido a que dichas plántulas son de crecimiento lento, en comparación con las hierbas que crecieron rápidamente y a la capacidad que tienen algunos arbustos de rebrotar, alcanzando en algunos casos alturas de hasta $2 \mathrm{~m}$ en los primeros 40 días, mientras que las plántulas sólo alcanzaban $15 \mathrm{~cm}$. Miller y Kauffman (1998) señalan que los brotes o retoños de los arbustos crecen rápidamente debido a sus reservas alimenticias almacenadas en las raíces, además son largos y delgados y su copa crece más rápidamente que las plántulas, las cuales tienen menores reservas en sus semillas que las de los brotes.

Otra ventaja de los brotes de arbustos en comparación con las plántulas, es la mayor superficie que abarca su sistema radical y la gran profundidad a que penetra en el suelo, característica relevante sobre todo en bosques secos donde el agua es un factor limitante. Debido a esto, Holl (1998) señala la importancia de reducir la competencia por hierbas y arbustos, ya que afectan de manera negativa el crecimiento de árboles jóvenes y plántulas, tanto en la regeneración natural como en proyectos de reforestación. Además, algunos arbustos podrían generar efectos alelopáticos sobre el crecimiento de las plántulas (Anaya 1976).

\section{Mortalidad}

El mayor porcentaje de mortalidad se presentó en los tratamientos sin control de la maleza, indicando una gran influencia de la misma sobre las plántulas de $T$. rosea.

Las plántulas que tuvieron mejor desarrollo lo reflejaron en un incremento de altura y diámetro, siendo más robustas, lo que les permitió una mayor capacidad de sobrevivir a condiciones adversas como la sequía. Steven (1994) informó que la supervivencia de plántulas de 
Quararibea asterolepis, Trichilia tuberculata y Tetragastris panamensis, en la Isla de Barro Colorado, Panamá, aumentó conforme incrementaban en edad y tamaño.

Al parecer, la principal causa de mortalidad de las plántulas de $T$. rosea fue la sequía o falta de humedad en el suelo, que aunado a la competencia con la maleza fue determinante. Esto coincide con lo expuesto por Kennard et al. (2002), quienes consideran que la supervivencia de las plántulas está estrechamente relacionada con la capacidad de poseer un amplio sistema radical que les proporcione una superficie mayor para la obtención de agua y nutrientes, así como una mayor profundidad en el suelo, especialmente en bosques secos donde el agua es estacionalmente limitada.

Los niveles de mortalidad en $T$. rosea se incrementaron conforme avanzó la época seca, las cifras de la última evaluación (Julio) reflejaron la condición de mayor sequía de los meses anteriores (Mayo, Junio). Estos resultados son congruentes con los informados por Gerhardt (1996), quien encontró que al final de la época de sequía todas las plántulas de Swietenia macrophylla (King) habían muerto en un bosque seco de Costa Rica y coinciden con lo señalado por varios autores respecto a que la principal causa de muerte de las plántulas en los bosques secos o con un marcado periodo seco, es debida a la desecación durante tal periodo (Steven 1994, Richards 1996, Kozlowski 2002).

\section{RESUMEN}

Evaluamos el efecto de los tratamientos de remoción de suelo y roza de vegetación sobre el establecimiento y desarrollo inicial de la regeneración natural de Tabebuia rosea (Bertold) DC. en una selva mediana subcaducifolia sometida a aprovechamiento maderable en la costa de Jalisco, México. Los tratamientos se aplicaron bajo la copa de árboles "semilleros" y se evaluó el número de plántulas emergidas y su desarrollo durante nueve meses. El tratamiento de remoción de suelo favoreció la emergencia de plántulas y su desarrollo inicial, mientras que el control de la vegetación competidora promovió un mayor crecimiento y menores porcentajes de mortalidad. Estos aspectos deben tomarse en cuenta para la regeneración natural de la especie después de la aplicación de las cortas con fines de aprovechamiento maderable y se sugiere incorporarlos a las técnicas silvícolas que actualmente se desarrollan en la zona.

Palabras clave: Selva mediana subcaducifolia, costa del Pacífico, regeneración natural, remoción de suelo, desarrollo inicial y mortalidad de plántulas, Tabebuia rosea.

\section{REFERENCIAS}

Ahlgren, C. 1974. Effects of fire on temperate forest: northcentral United States, p 279-391. In T.T. Kozlowski $\&$ C.E. Ahlgren. Fire and Ecosystems. Academic, Nueva York, EEUU.

Anaya, L. 1976. Alelopatía en plantas superiores: diferencias entre el efecto de la presión osmótica y los alelopáticos sobre la germinación secundaria de una zona cálido húmeda de México, p. 388-427. In A. Gómez-Pompa, C. Vázquez Yánez, S. Del Amo R. \& A. Butanda C. Regeneración de Selvas. CECSA, México, DF, México.

Ángeles, G. \& P. Sakimoto. 1999. Emergence disappearance process and mortality factors of current year seedlings of Abies firma in a natural Abies-Tsuga forest, Wakayama. For. Res. Kyoto. 71: 27-33.

Ángeles, G., A. Velázquez, J. Vargas, H. Ramírez \& M. Musálem. 1997. Efecto del manejo de la vegetación en algunas variables de crecimiento de la repoblación natural de un rodal de Pinus patula en el estado de Hidalgo (México). Invest. Agr. Sist. Recur. For. 6: 119-131.

Anónimo. 1994. Secretaria de Agricultura y Recursos Hidráulicos. Inventario Forestal Periódico del Estado de Jalisco, México. Gobierno Estatal, Jalisco, México. 78 p.

Anónimo. 1999. S-Plus 2000 modern statistics and advanced graphics (user's guide). Mathsoft, Massachusetts, EEUU. 557 p.

Augspurger, C. 1984. Seedling survival of tropical tree species: interactions of dispersal distance, light-gaps, and pathogens. Ecology 65: 1705-1712.

Beland, M., E. Agestam, P. Pekö, P. Gemmel \& U. Nilsson. 2000. Scarification and seedfall affects natural regeneration of Scots pine under two shelterwood densities and a clear-cut in Southern Sweden. Scand. J. Forest Res. 15: 247-255.

Cochran, W.G. 1998. Técnicas de muestreo. CECSA, México, DF, México. 513 p.

Finegan, B. 1984. Forest succession. Nature 312: 109-114. 
Gerhardt, K. 1996. Germination and development of show mahogany (Swietenia macrophylla King) in secondary tropical dry forest habitats in Costa Rica. J. Trop. Eco. 12: 275-289.

Hardwick, K., J. Healey, S. Elliott \& N. Garwood. 1997. Understanding and assisting natural regeneration processes in degraded seasonal evergreen forest in northern Thailand. Forest Eco. Manag. 99: 203-214

Hartshorn, S. 1980. Neotropical forest dynamics. Biotropica 12 (Supl.): 23-30.

Hawley, R. \& D. Smith. 1972. Silvicultura práctica. Trad. por J. Terrada. Omega, Barcelona, España. 544 p.

Holl, K.D. 1998. Effects of above -and below- ground competition of shrubs and grass on Calophyllum brasiliense (Camb.) seedling growth in abandoned tropical pasture. Forest Eco. Manag. 109: 187-195.

Infante, S.G. \& G.P. Zárate de Lara. 1990. Métodos estadísticos un enfoque interdiciplinario. Trillas, México, DF, México. 643 p.

Karlsson, C. \& G. Örlander. 2000. Soil scarification shortly before a rich seed fall improves seedling establishment in seed tree stands of Pinus sylvestris. Scand. J. Forest Res. 15: 256-266.

Kennard, D., K. Guold, F. Putz, T. Fredericksen \& F. Morales. 2002. Effect of disturbance intensity on regeneration mechanisms in a tropical dry forest. Forest Ecol. Manag.162: 197-208.

Kozlowski, T. 2002. Physiological ecology of natural regeneration of harvested and disturbed forest stand: implications for forest management. Forest Ecol. Manag. 158: 195-221.

Lamprecht, H. 1990. Silvicultura en los trópicos. Cooperación Técnica Alemana, Berlín, Alemania. $335 \mathrm{p}$.
Lowery, R., C. Lambeth, M. Endo \& M. Kane. 1993. Vegetation management in tropical forest plantations. Can. J. Forest Res. 23: 2006-2014.

Macario, M. 1991. La repoblación natural en una selva mediana subperenifolia en Quintana Roo bajo aprovechamiento forestal. Tesis de Maestría, Colegio de Postgraduados, Montecillo, México. 131 p.

Miller, P. \& J. Kauffman. 1998. Seedling and sprout response to slash and burns agriculture in a tropical deciduous forest. Biotropica 30: 538-546.

Miranda, F. \& E. Hernández X. 1963. Los tipos de vegetación de México y su clasificación. Bol. Soc. Bot. Méx. 28: 29-179.

Molofsky, J. \& C. Augspurger. 1992. The effect of leaf litter on early seedling establishment in a tropical forest. Ecology 73: 68-77.

Musálem, M. 1984. Effect of environmental factors on regeneration of Pinus montezumae Lamb., in temperate forest of México. Ph. D. Dissertation, Yale University, Connecticut, EEUU. 262 p.

Oliver, C.D. \& B.C. Larson. 1990. Forest stand dynamics. McGraw-Hill, Nueva York, EEUU. 467 p.

Richards, P. 1996. The tropical rain forest: an ecological study. Cambridge, Cambridge, Inglaterra. 575 p.

Steven, D. 1994. Tropical tree seedling dynamics: recruitment patterns and their population consequences for tree canopy species in Panama. J. Trop. Ecol. 10: 369-383.

Uhl, C. 1987. Factors controlling successions following slash and burn agriculture in Amazonia. J. Ecol. 75: 377-407.

Velázquez, M. 1984. Estudio de algunos factores que influyen en la regeneración natural de Pinus hartwegii Lindl., en Zoquiapan, México. Tesis de Maestría, Colegio de Postgraduados, Montecillo, México. 123 p. 
